http://jmscr.igmpublication.org/home/

ISSN (e)-2347-176x ISSN (p) 2455-0450

crossref DOI: https://dx.doi.org/10.18535/jmscr/v8i7.57

Journal Of Medical Science And Clinical Research

\title{
Comparing efficacy of Topiramate and Valproate in migraine prophylaxis in rural Bengal -A Randomized Controlled Trial
}

\author{
Authors \\ Abhijit Das ${ }^{1}$, Ratul Banerjee ${ }^{2 *}$, Shankar Prasad Nandi ${ }^{3}$, Ananya Mandal ${ }^{4}$ \\ ${ }^{1} \mathrm{MD}$ (Pharmacology), Professor and Head, Department of Pharmacology, Burdwan Medical College \& \\ Hospital \\ ${ }^{2}$ MD (Pharmacology), MO SUPY, Salt Lake Subdivisional Hospital \\ ${ }^{3}$ DM (Neurology), Assistant Professor, Department of Neurology, Bankura Sammilani Medical College \& \\ Hospital \\ ${ }^{4} \mathrm{MD}$ (Pharmacology), Associate Professor, Department of Pharmacology, NRS Medical College \& Hospital \\ *Corresponding Author \\ Ratul Banerjee
}

\begin{abstract}
Migraine is second most common cause of headache which is responsible for reduction in the quality of life affects near about 15\% of women and 6\% of men over a period of 1 year.1 Affect boys and girls in similar fashion in prepubescent age group, but girls are affected more than boys after that with a rise of incident in fourth decade of life. Topiramate and sodium valproate are the two widely accepted and approved anti-epileptics used for treatment of migraine prevention. Head to head studies are few with these drugs and we have taken MIDAS Score with reduction of frequency and duration of migraine headache as primary and European quality of life index with EQ-VAS score for quality of life assessment, all these parameters are not founded in any study. A randomized control trial was done at Bankura Sammilani Medical College with population from rural Bengal, data was taken and detail and appropriate statistical analysis was done with appropriate software. Both the drugs were found very much effective for migraine prophyalxis and improving quality of life.

Keywords: Migraine, Valproate, Topiramate.
\end{abstract}

\section{Introduction}

Migraine is one type of headache, descripted as recurring syndrome of headache associated with other certain neurological dysfunction in varying admixtures. ${ }^{1}$ Migraine is a common neurological disease that often hampers daily life. It is the second most common cause of headache, and it is most common headache related, and indeed neurologic cause of disability in the world, affects near about $15 \%$ of women and $6 \%$ of men over a period of 1 year. ${ }^{1}$ Many epidemiological studies have documented its high prevalence and socioeconomic and personal impacts. In the Global Burden of Disease Study2010 (GBD2010), it was ranked as the third most prevalent disorder in the world. In GBD2015, it was ranked thirdhighest cause of disability worldwide in both males and females under the age of 50 years. Migraine has a one-year prevalence of $12 \%$ in the general population, it consist of $18 \%$ in case of 
women and $6 \%$ of men., ${ }^{2,3}$ Migraine has similar distribution in prepubescent boys and girls. At puberty, the migraine incidence increases sharply in both boys and girls, but preferentially it affects girls much more. Peak migraine prevalence for both sexes occurs in the fourth decade of life in the time period approximately $24 \%$ of women and $7 \%$ of men have migraine. ${ }^{2}$ Numerous studies have confirmed that migraineurs experience a poor quality of life and diminished feelings of well-being even during the pain-free intervals. Studies performed in different countries, either on individuals from the general population or on patients from headache clinics, reveal that migraine is associated with significantly lower scores on various health-related quality-of-life rating scales, regardless of age, gender, or socioeconomic status. ${ }^{4-7}$ International evidencebased guidelines from the US Headache Consortium $^{8}$ and the European Federation of Neurological Societies (EFNS) ${ }^{9}$ have focused on the circumstances warranting preventive treatment in migraineurs. Topiramate and sodium valproate are the two widely accepted anti-epileptics used for treatment of migraine prevention. Topiramate is a sulfamate-substituted monosaccharide that is FDA-approved as initial monotherapy (in patients at least 10 years old) and as adjunctive therapy (for patients as young as 2 years) for focal-onset or primary generalized tonic-clonic seizures, for Lennox-Gastaut syndrome in patients 2 years of age and older, and for migraine headache prophylaxis in adults. ${ }^{10}$

The recommended total daily dose of topiramate for prophylaxis of migraine is $100 \mathrm{mg}$ a day administered in 2 divided doses. The drug should be slowly titrated to this dosage over 4 weeks, starting as low as $25 \mathrm{mg}$ a day in week 1. One Class I placebo-controlled study showed a lower 28-day frequency of migraine headaches versus placebo (3.31 versus 3.81). In the regulatory trials, $100 \mathrm{mg}$ was the optimal dose for prevention. ${ }^{11-}$ ${ }^{2}$ Valproate products are FDA-approved drugs to treat seizures, and manic or mixed episodes associated with bipolar disorder (manic-depressive disorder), and to prevent migraine headaches. In adult Valproate is recommended as 400-600 mg /day for migraine prophylaxis. Very few head to head studies are present using these two drugs as migraine prophylaxis. We have found no study regarding EQ-5D-5L scoring improvement with drug for migraine prophylaxis, which scoring has described five separate parameters for measuring patient's health status. The objective of the study was to assess comparative efficacy of Sodium valproate $(500 \mathrm{mg})$ and Topiramate $(100 \mathrm{mg})$ in reducing migraine headache as measured by comparing change of Frequency (attack per month), MIDAS score, duration of headache and to assess comparative efficacy of investigational drugs in two aforesaid treatment groups in improving physical quality of life and the impact on general health related quality of life as elicited by comparing change of EQ-5D-5L Questionnaire and EQ-VAS score.

\section{Materials and Method}

It was an Interventional study designed as Prospective, randomized, parallel group, openlabel, two arm trial. The study done at, Room no 1 in the outpatient department in Department of Neurology, Bankura Sammilani Medical College at Lokepur, Bankura.

\section{Inclusion Criteria}

- Adult and adolescent (age > 12 years) patients diagnosed with migraine according to the $3^{\text {rd }}$ edition (beta version) of International Classification of Headache Disorders (ICHDIII) criteria of International Headache Society (IHS),

- Patients, with presence of an indication for prophylactic treatment (intolerable headache attacks that were either debilitating or resulted in significant loss of daily function

- Frequent attacks ( $\geq 4$ attacks per month)

\section{Exclusion Criteria}

- Other causes of headache

- Major illness

- Any co morbidity 
- Pregnant and lactating mother

- Any known allergies to the study drugs We have done the study for 18 months, from February 2017 to July 2018.

Sample size were calculated by the formula $(\mathrm{z} \alpha+$ $\mathrm{z} \beta)^{2} \mathrm{x}\left(\sigma_{1}+\sigma_{2}\right) /(\mu 1-\mu 2)^{2}$ as minimum 72 including $20 \%$ dropout, where $\mathrm{Z} \alpha$ is $\alpha$ error $=1.96, \mathrm{Z} \beta$ is $\beta$ error $=0.84, \sigma_{1^{-}}$standard deviation of first group, $\sigma_{2-}$ standard deviation of second group, both are same as taken from same population and $(\mu 1-\mu 2)$ is deviation of MIDAS score from baseline expected after treatment $=5$.

We have studied reduction in the frequency, which is no. of headache per month, reduction in the duration of headache and MIDAS score. The quality of life was assessed using to EQ-5D-5L questionnaire. It contains 5 headings and each heading contains 5 questions, each was coded in Excel sheet against 1-5 score. A greater score indicates poor quality of life. Each heading analyzed separately. Another scoring done by the EQ VAS that is a visual analogue scale suggested how bad is the patient's health for that day. It is a subjective criterion. Score ranges from 0-100. 0 means worst and 100 means the best one can imagine.

We have done the study for 18 months, from February 2017 to July 2018, included all patients who met our inclusion and exclusion criteria. This study was done following the principles of the Declaration of Helsinki for study on human subjects. This study was conducted only after obtaining proper written approval from the Institutional Ethics Committee. Written informed consents were taken from every study patient or their legal representatives. It was registered in Clinical Trial Registry India (CTRI) under Indian Council for Medical Research (ICMR), Government of India. The registration number is

\section{CTRI/2017/07/009074.}

After arrival of the patients at neurology OPD, consultant neurologist examined them. Those diagnosed by him, other cause of headache were excluded by history, clinical or relevant imaging (CT scan) and the patients were passed through a

\section{printed}

preformed

validated

MIDAS questionnaire. Those who scored $\geq 5$, were taken as having migraine need prophylactic treatment. At baseline level, a MIDAS score assessment was done. Also a baseline assessment of EQ-5D-5L score \& EQ-VAS score was done. He was also asked to maintain a migraine diary to note the date of attack of headache during every month, and last month total days were collectively noted as frequency. These were accompanied by necessary baseline laboratory investigations, i.e. Complete Blood Count, Blood sugar, Liver function test, Urea, Creatinine, Sodium, Potassium estimation, 12 lead ECG etc. Now this patients were randomly allocated into 2 groups using pre-set computer generated random numbers and were prescribed the following drugs by consultant neurologist.

Group V = Valproate $500 \mathrm{mg}$ Once daily dose Group $\mathbf{T}=$ Topiramate $50 \mathrm{mg}$ twice daily dose No adjuvant medicine were given as comorbidities were excluded to avoid possible drug interaction but for controlling the attack in some case abortive concurrent medication were prescribed as and when necessary.

Patients were followed up for two visits, after 12 weeks and 24 weeks interval from the day of starting treatment. On each visit, assessment was done by Frequency, MIDAS score, EQ-5D-5L score and EQ-VAS score. At the end of follow up after 12 weeks and 24 weeks, all baseline investigations were repeated. The drop outs or withdrawal if any along with reasons for the same were recorded. Data was collected in a specially designed case record form (CRF) by conducting a personal interview with each patient during the clinic visit. Data were entered in Microsoft Excel $\&$ checked for accuracy. Data were analyzed with the help of SPSS version 22 and Graph Pad Prizm version 5. Normalcy was checked by the Kolmogorov-Smirnov test and Shapiro-Wilk test. For estimating change in Frequency, MIDAS, EQ5D-5L \& EQ-VAS score within a particular group from baseline, we used Friedman's ANOVA, Repeated measure ANOVA followed by Dunn's 
$\&$ Wilcoxon match pair sign rank test post-hock analysis. Whereas for estimating difference between different treatment groups at different follow up visit we used Mann whitney $U$ and Unpaired t test followed by Dunn's \& Tukey's post-hock analysis. All analyses were two sided. P value less than 0.05 was taken as significant.

\section{Result}

During entire study period, we encountered a total of 108 patients, of which 30 patients did not meet the inclusion \& exclusion criteria. Hence, 78 patients were enrolled. But 10 patients were lost to follow up. Final analysis was done on 68 patients.

Females were majority $(69.11 \%)$ outnumbering the males to a great extent. Maximum numbers of patients $(63.37 \%)$ belonged to age group of 20-40 years (Table 1). Other two groups <20 yr and $>40$ yr almost share same percentage (Figure 1).

The age distribution data was parametric according to Kolmogorov-Smirnov test. Then we performed Unpaired $\mathrm{t}$ test to find out any significant difference in age distribution between the groups. The $\mathrm{P}$ value was found to be 0.398 . No post hoc test was required.

We used three principal variables- to see decrease in Headache Frequency (that is headache days per month), MIDAS Score and EQ-5D-5L score to see in improvement of quality of life. EQ-5D-5L has subgroup of Mobility, Self-care, Usual activity, Pain/ Discomfort and Anxiety/Depression which have level coded from 1-5. 1 signifies best and 5 signifies worst outcome.

We performed Unpaired $t$ test for headache frequency to find out any significant difference between groups at baseline as the data parametric. In group $\mathrm{V}$ and in group $\mathrm{T}$ Mean $\pm \mathrm{SD}$ was $9.054 \pm 2.107 \& 7.839 \pm 2.410$ and $p$ value was 0.0874 .We performed Mann whitney $U$ to find out any significant difference between groups for MIDAS score and at baseline as the data was non-parametric. Mean \pm SD was 19.14 \pm 3.17 $\& 18.29 \pm 3.60$ respectively. P value was 0.0678 .
We have analysed EQ-5D-5L parameter separately and in group Valproate and in group Topiramate and the result we found Mean \pm SD was in case of Mobility 3.378 $\pm 0.892 \& 3.355 \pm 1.33$ with $\mathrm{p}$ value 0.2441 , in case of Self-care $3.56 \pm 0.8$ $\& 3.226 \pm 1.283$ with $\mathrm{p}$ value 0.1538 , in case of Usual activity $3.216 \pm 1.004 \& 3.677 \pm 0.944$ with $p$ value 0.1781 , in case of Pain/ Discomfort $3.378 \pm 0.728 \& 3.387 \pm 1.022$ with $p$ value 0.5643 and in case of Anxiety/Depression it was $3.622 \pm 0.681 \& 3.032 \pm 1.251$ with $p$ value 0.0505 . So no significant difference was found at baseline. We have also studied EQ-VAS (Visual Analogue Scale) Score for intensity and duration of headache also. In EQ-VAS 0-100 marking is present, where 0 means the worst and 100 means the best health one can imagine. Duration of headache was expressed in hours. Mean \pm SD was $35.023 \pm 8.476$ and $40.36 \pm 8.227$ in Group V and $\mathrm{T}$ respectively and $\mathrm{p}$ value was 0.0951 at the baseline, when we studied EQ -VAS and Mean \pm $\mathrm{SD}$ in case of duration of headache was $11.76 \pm 3.427$ and $11.39 \pm 2.472$ and $p$ value was 0.1105 . So, in both occasion we found nonsignificant result.

Change of Headache Frequency done within group V (Repeated Measure ANOVA) followed by Wilcoxon match pair post-hoc test, and Mean \pm SD was 9.054 $\pm 2.107, \quad 6.027 \pm 1.236$ and $2.649 \pm 1.317$ respectively from baseline to follow up and $p$ value was very much significant $<0.0001$. It was significant in both the follow up. Same observation with group $\mathrm{T}$ Mean \pm SD was $7.839 \pm 2.41, \quad 5.419 \pm 1.232$ and $2.097 \pm 1.274$ respectively from baseline to follow up and $\mathrm{p}$ value was very much significant $<0.0001$ (Table 2 $\&$ Figure 2). It was significant in both the follow up.We performed Unpaired t test to find out any difference in mean Headache Frequency between the groups over three follow ups and found the result in first follow-up p value was 0.0658 and in second follow-up p value was 0.1284 . So, both the drugs were equally effective in headache frequency reduction. 
Change of MIDAS Score done within group V (Friedman ANOVA) followed by Dunn's post-hoc test, and Mean \pm SD was 19.14 \pm 3.172, $10.34 \pm 2.599$ and $5.94 \pm 1.966$ respectively from baseline to follow up and $\mathrm{p}$ value was very much significant $<0.0001$. It was significant in both the follow up. Same observation with group T Mean \pm SD was 18.29 $\pm 3.607,10.45 \pm 3.009$ and $5.387 \pm 2.261$ respectively from baseline to follow up and $\mathrm{p}$ value was very much significant $<0.0001$ (Table $3 \&$ Figure 3). It was significant in both the follow up. We performed Mann-whitney $U$ test to find out any difference in mean Headache Frequency between the groups over three follow ups and found the result in first follow-up p value was 0.7274and in second follow-up $p$ value was 0.8441 . So, both the drugs were equally effective in MIDAS Score reduction.

After doing an exhaustive statistical test on the 5 parameter of EQ-5D-5L data, we came to the conclusion that both the drugs are very much effective and significant change (0.05) noted in Valproate and Topiramate group from baseline to first follow up and baseline to second follow up. But no significant changes after that in second follow up than first follow up in all the parameter (Table4).

Change of EQ-VAS done within group V (Repeated Measure ANOVA) followed by Wilcoxon match pair post-hoc test, and Mean was $35.03,51.94$ and 68.48 respectively from baseline to follow up and $\mathrm{p}$ value was very much significant. Same observation with group T. Mean was 40.36, 54.39 and 70.09 respectively from baseline to follow up and $p$ value was very much significant (Figure 4). It was significant in both the follow up. No significant change in between groups was observed as tested by unpaired t test. Change of headache duration within group $\mathrm{V}$ (Friedman ANOVA) followed by Dunn's post-hoc test, and Mean was 11.76, 6.703 and 3.514 respectively from baseline to follow up and $\mathrm{p}$ value was very much significant. Same observation with group T. Mean was 11.39, 6.742 and 3.097 respectively from baseline to follow up and $\mathrm{p}$ value was very much significant (Figure 5). It was significant in both the follow up. No significant changes in between groups were observed as tested by Mann whiteney $\mathrm{U}$ test.

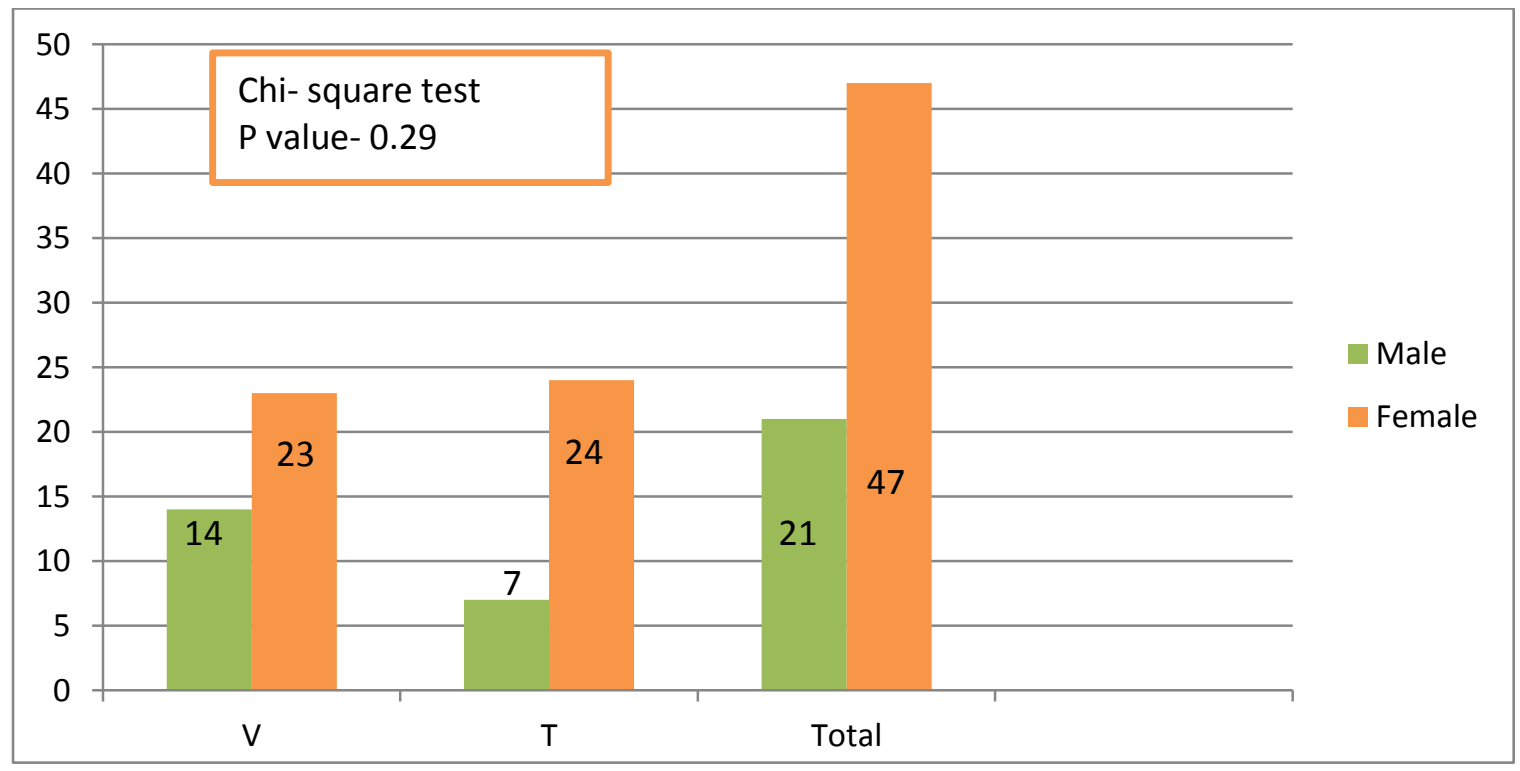

Figure 1 Demographic characteristic

Table 1 Age distribution

\begin{tabular}{|l|c|c|}
\hline Group & Mean (Yrs) & SD (Yrs) \\
\hline V & 31.35 & 9.025 \\
\hline $\mathbf{T}$ & 28.35 & 9.138 \\
\hline
\end{tabular}




\section{JMSCR VoI||08||Issue||07||Page 357-366||July}

Table 2 Reduction of headache frequency group wise

\begin{tabular}{|l|c|c|}
\hline & $\mathbf{V}$ & $\mathbf{T}$ \\
\hline Baseline & 9.054 & 7.839 \\
\hline 1st Visit & 6.027 & 5.419 \\
\hline 2nd Visit & 2.649 & 2.097 \\
\hline
\end{tabular}

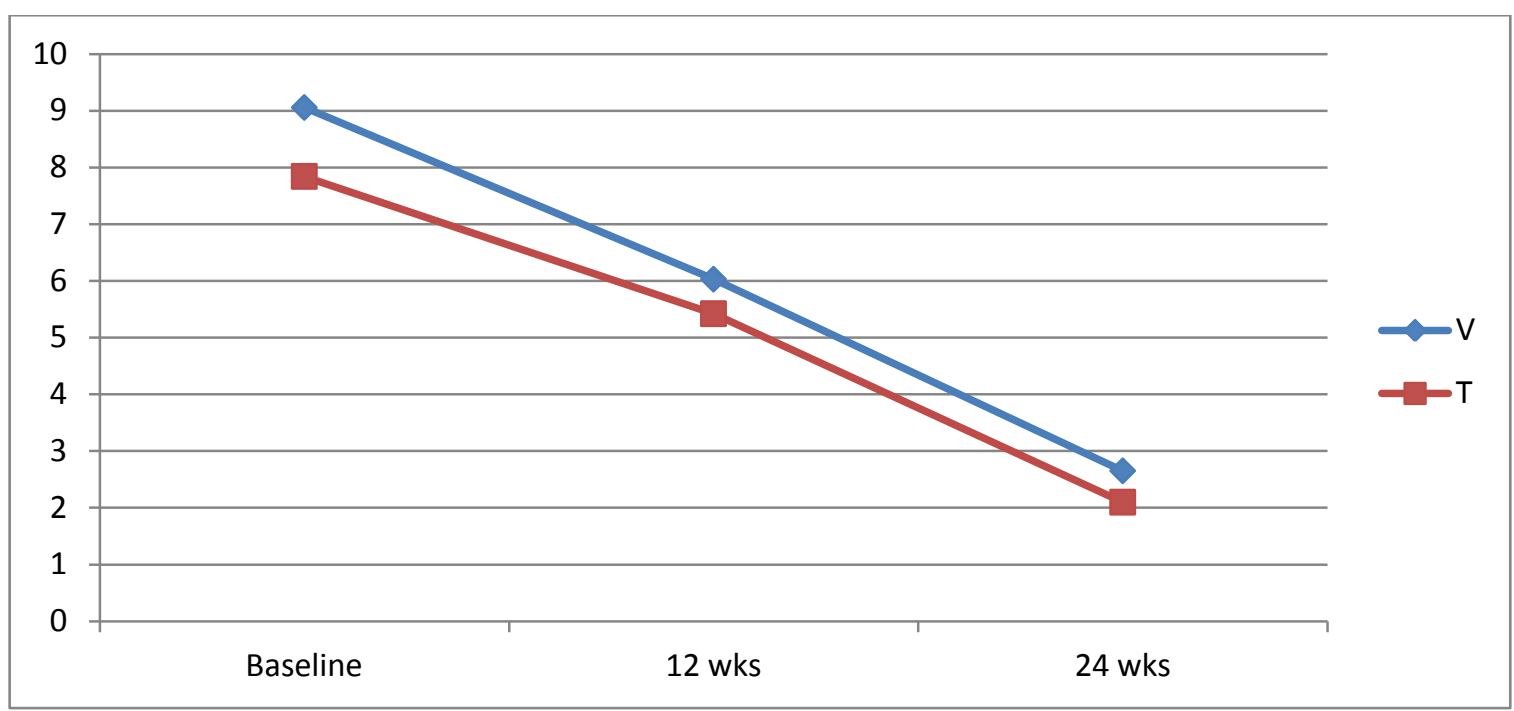

Figure 2 Reduction of headache frequency group wise

Table 3 Reduction in MIDAS Score group wise

\begin{tabular}{|l|c|c|}
\hline & $\mathbf{V}$ & $\mathbf{T}$ \\
\hline Baseline & 19.14 & 18.29 \\
\hline 1st Visit & 10.54 & 10.45 \\
\hline 2nd Visit & 5.541 & 5.387 \\
\hline
\end{tabular}

Figure 3 Reduction of headache MIDAS Score group wise

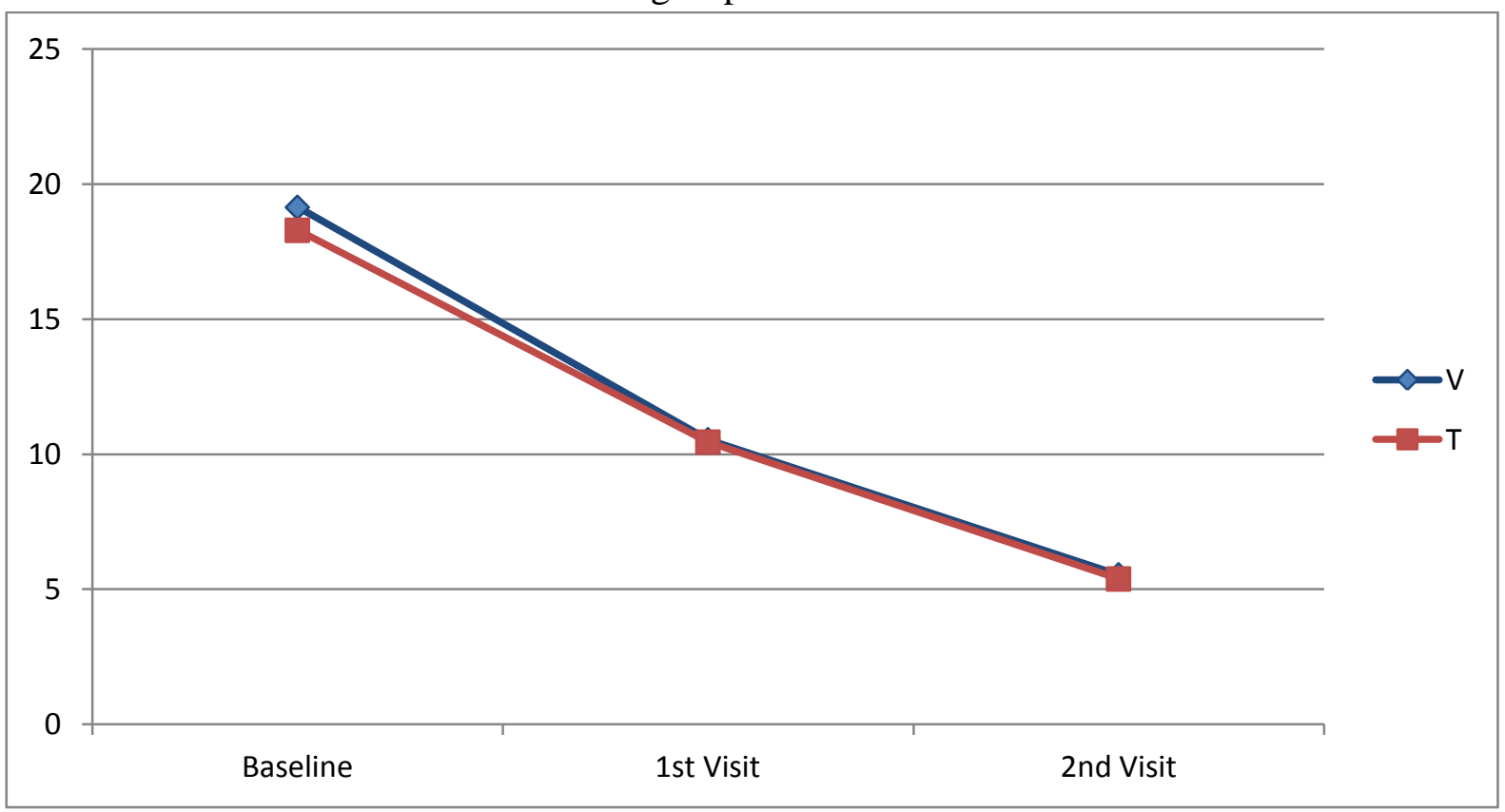


Table 4 Change of quality of life

\begin{tabular}{|l|c|c|}
\hline & Post hoc test & Group shows change \\
\hline $\begin{array}{l}\text { Mobility / Self- care / Usual activity / Pain } \\
\text { \&Discomfort / Anxiety \& Depression }\end{array}$ & Within group & V \& T \\
\cline { 2 - 3 } & Between group & No change \\
\hline
\end{tabular}

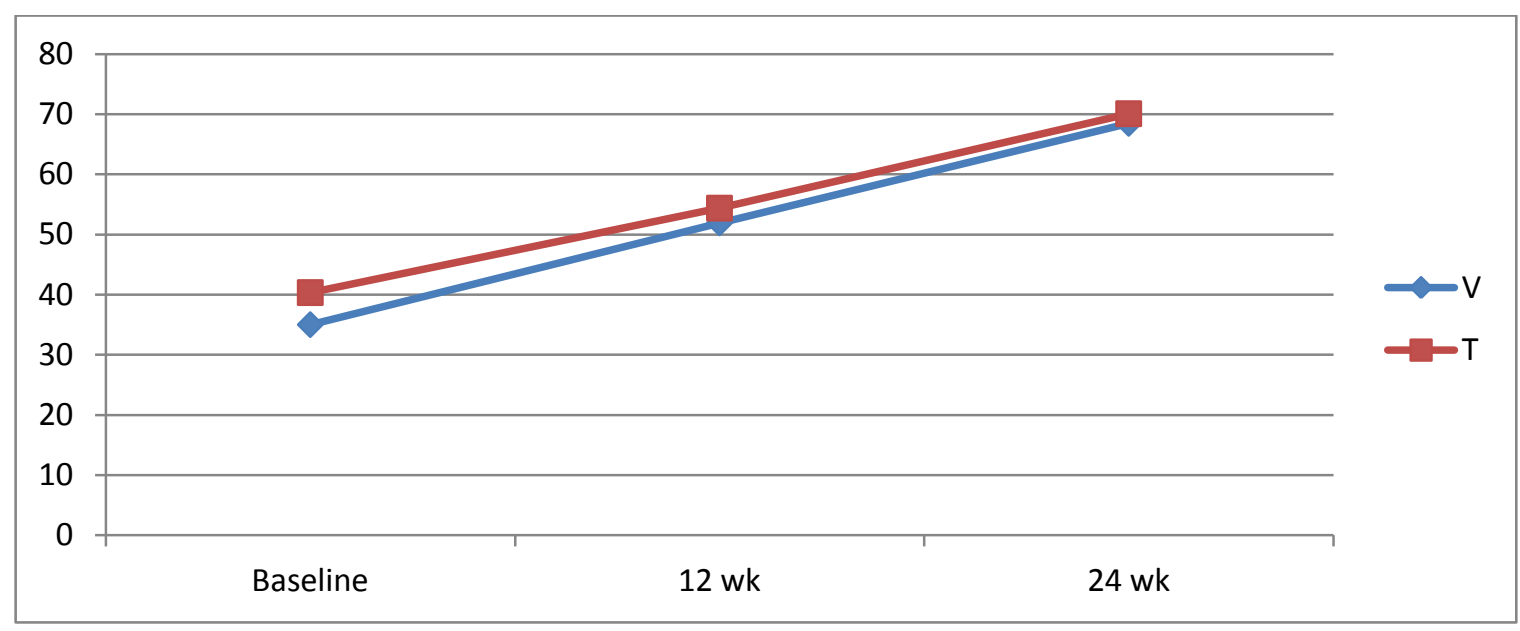

Figure 4 Changes in EQ-VAS Score

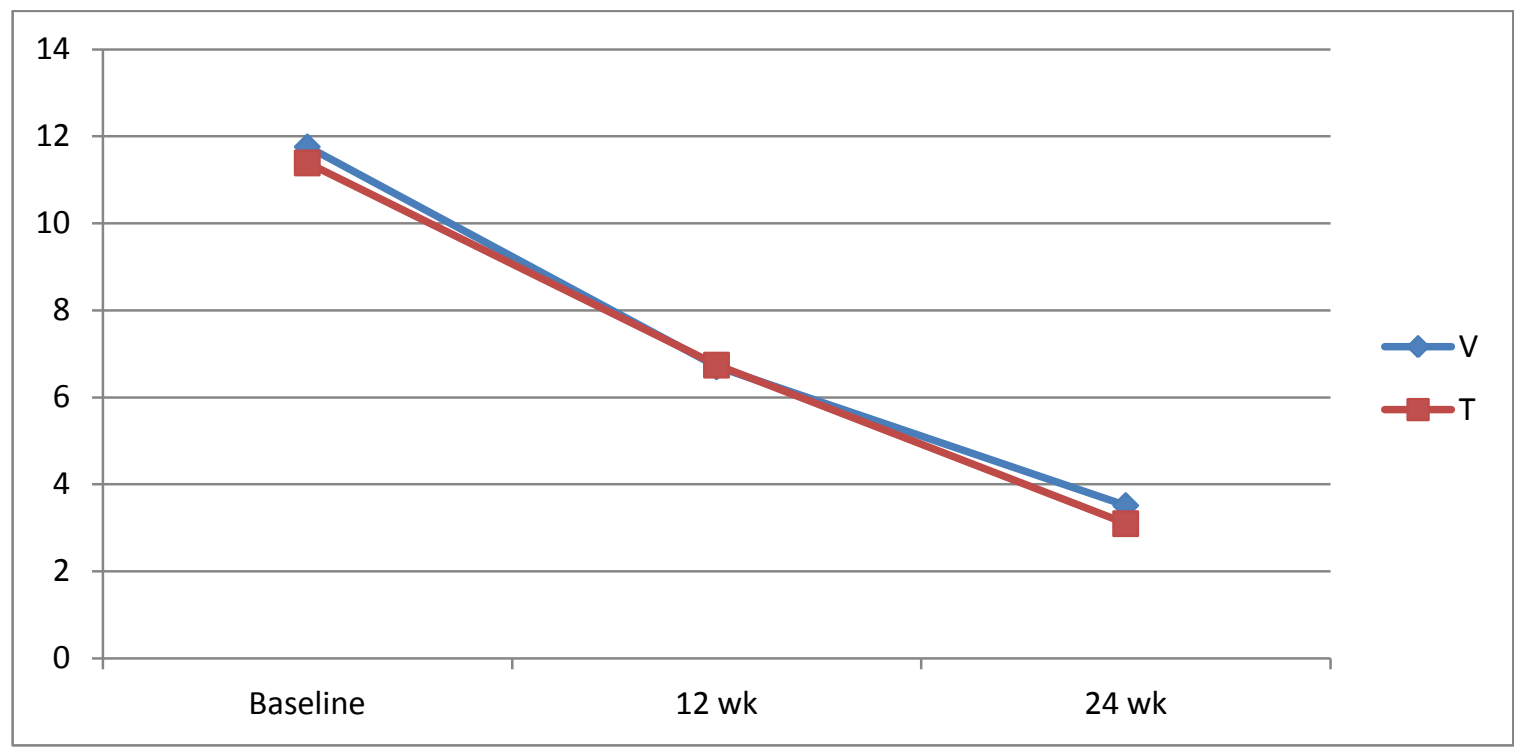

Figure 5 Reduction in headache duration

\section{Discussion}

In spite of having a number of treatment options, the management of migraine has not reached a desired level. As one has to take medicines for prolonged duration \& long term ADRs often precludes uninterrupted treatment. Beside this, the poor quality of life often hamper the patients' compliance and treatment adherence. It is thus of utmost important to find out the treatment with a drug (s) with optimum balance between efficacy and safety. We have made extensive search through published literature, but failed to find any study which includes these drugs with all this parameter for head to head comparison for treatment of migraine in OPD setting. Though fewer study is there with Valproate and Topiramate. Though anticonvulsants are also well tolerated, and they cause fewer adverse effects ${ }^{13-4}$ Topiramate (TPM) and valproic acid (VPA) are the most important treatment options for migraine and are now approved for migraine prevention in several countries. ${ }^{15-6}$ Meta-analysis of efficacy of 
topiramate in migraine prophylaxis Yiyi Guo et al by searching the Medline database, EMbase, Cochrane Library and China National Knowledge Infrastructure database for articles published between January 1995 and May 2011, using the key words "migraine", "topiramate", and "prophylaxis" found eight randomized controlled trials were appropriate, and had available data. The meta analysis results revealed that topiramate (100 or $200 \mathrm{mg} / \mathrm{d}$ ) was more effective than placebo in responder rate $(\mathrm{OR}=2.97,95 \%$ confidence interval (CI): 2.17-4.08, P < 0.01; OR $=2.35$, 95\%CI: 1.77-3.12, P < 0.01). Topiramate $(100 \mathrm{mg} / \mathrm{d})$ was more effective than placebo in terms of the change in mean monthly migraine days (MD: $-1.14,95 \% \mathrm{CI}:-1.69$ to $-0.59, \mathrm{P}<$ 0.01). ${ }^{17}$

Kinze S et al, 2001 at Department of Neurology, Charité Clinic, Humboldt University of Berlin have done a study with valproic acid doses within a range of 500 to $1500 \mathrm{mg}$ per day for migraine prophylaxis as valproic acid serum levels less than $50 \mathrm{microg} / \mathrm{mL}$ (group 1) and those with serum levels greater than $50 \mathrm{microg} / \mathrm{mL}$ (group 2) on 52 patients and found that the frequency of migraine attacks was significantly reduced in group 1 from $3.5+/-0.9$ to $2.0+/-0.9$ attacks per month. Migraine headache days also decreased $(6.4+/-$ 3.5 to $4.6+/ 2.9$ days per month). The outcome of group 1 (low serum level) was significantly better than that of group 2 with respect to both parameters $(\mathrm{P}<.05) .^{18}$

In another study at baseline the 2 groups had similar numbers of days with headache and mean MIDAS scores. At the end of the treatment period, a significant reduction in 30-day headache frequency with respect to baseline $(\mathrm{P}<0.00001)$ and a significant reduction in MIDAS scores $(\mathrm{P}$ $<0.00001)$ were recorded in both groups. There were no significant differences in beneficial effects between the 2 drugs. ${ }^{19}$ A randomized, double-blind, parallel-group clinical trial done on 56 patients with. Topiramate and valproate were administered at $50 \mathrm{mg} /$ day and $400 \mathrm{mg} /$ day, respectively, during the follow-up period.
Frequency, intensity, duration, associated symptoms with headaches, analgesics use, as well as drugs' side effects were studied. Participants completed MIDAS and HIT-6 questionnaires before and after treatment. Frequency, intensity, and duration of migraine headaches as well as MIDAS score and symptomatic medications decreased significantly between repeated followup visits in both groups. Responder rate for patients treated with topiramate and valproate were $71.6 \%$ and $64.3 \%$, respectively, and the difference between the two groups was not statistically significant. The reduction of headache severity in the topiramate group was significantly more than that in the valproate group $(\mathrm{p}=.027)$. Afshari D, Rafizadeh S, Rezaei M.

R. Bavarsad et $\mathrm{l}^{20}$ done a study with The effects of middle dose of topiramate $(50-75 \mathrm{mg}$ ) and sodium valproate $(400-600 \mathrm{mg}$ ) in the prevention of migraine headache with 73 patients and found MIDAS score in Topiramate group reduced more than the group receiving valproate sodium, which indicates changes, was statistically significant in both groups before treatment.

In our study we have done treatment with $100 \mathrm{mg}$ Topiramate and $500 \mathrm{mg}$ Sodium valproate and found that both the drug was very much effective in reducing MIDAS Score, headache duration, frequency and quality of life over two follow-up but no significant changes were noted between the group which was no difference with the previous studies.

\section{Conclusion}

In this open-label, parallel group, 24 weeks (first follow up12 wk and second follow up 24 wk), interventional study we found that Valproate and Topiramate both were highly effective in migraine prophylaxis. There were no differences between the groups within first and second follow-up. Migraine frequency and duration was reduced, as well as the migraine associated disability assessed by MIDAS score. Quality of life was also improved significantly in those groups from baseline which may be due to both pain reduction 
and also improve in usual activity, mobility and anxiety/depression with those two groups of drugs.

\section{Acknowledgement}

Authors would like to thank to Dr. P Barma, H.O.D. Department of Neurology and Dr. S.M. Naser, H.OD. Department of Pharmacology and also to Associate Professor Dr. Sonai Mandal and Associate Professor Dr. Tanmoy Gangopadhyay for their co-operation in present work and also grateful to all non-medical staffs of Neurology OPD and Pharmacology Department of Bankura Sammilani Medical College for their help in present work. Authors are also thankful to all PGTs and other faculty of Pharmacology Department of the same institution.

Work Attributed to: Department of Pharmacology and Department of Neurology, Bankura Sammilani Medical College \& Hospital

Sources of Support: Department of Pharmacology and Department of Neurology, Bankura Sammilani Medical College \& Hospital

Funding: No Funding Sources

Conflict of Interest: None Declared

\section{References}

1. Kasper DL, Fauci AS, Hauser SL, Longo DL, Jameson JL, Loscalzo J. Harrison's Manual of Internal Medicine. 19. 1. New York: McGraw Hill Education; 2016.215.

2. Lipton RB, Bigal ME, Diamond M, Freitag F, Reed ML, Stewart WF; AMPP Advisory Group. Migraine prevalence, disease burden, and the need for preventive therapy. Neurology. 2007;68(5):343-9.

3. Rasmussen BK. Epidemiology of headache. Cephalalgia.1995;15(1):44-67.

4. Osterhaus JT, Townsend RJ, Gandek B, Ware JE Jr. Measuring the functional status and well-being of patients with migraine headache. Headache. 1994 Jun; 34(6):337-43.
5. Monzón MJ, Láinez MJ. Quality of life in migraine and chronic daily headache patients. Cephalalgia. 1998;18(9):638-43.

6. D'Amico D, Usai S, Grazzi L, et al. The impact of primary headaches on patients' lives: Italian experience with the MIDAS and the SF-36 questionnaires. Headache Care. 2004;1:123-8.

7. Lucas C, Géraud G, Valade D, Chautard MH, Lantéri-Minet M. Recognition and therapeutic management of migraine in 2004, in France: results of FRAMIG 3, a French nationwide population-based survey. Headache. 2006; 46(5):715-25.

8. Silberstein SD.P ractice parameter: evidence-based guidelines for migraine headache (an evidence-based review): report of the Quality Standards Subcommittee of the American Academy of Neurology. Neurology. 2000; 55(6):754-62.

9. Evers S, Afra J, Frese A, Goadsby PJ, Linde M, May A, Sándor PS.EFNS guideline on the drug treatment of migraine - report of an EFNS task force.Eur J Neurol. 2006;13(6):560-72 .

10. Brunton LL, Hilal-Dandan R, Knollmann BC. Goodman Gilman's The Pharmacological Basis Of Therapeutics. 13.New York: McGraw Hill;2018.318-9.

11. Brandes JL, Saper JR, Diamond M, et al. Topiramate for migraine prevention: a randomized controlled trial. JAMA. 2004;291(8):965-73 12. Diener HC, Tfelt-Hansen P, Dahlof $\mathrm{C}$, et al. Topiramate in migraine prophylaxis results from a placebo-controlled trial with propranolol as an active control. J Neurol. 2004;251(8):943-50.

12. Chronicle E, Mulleners W. Anticonvulsant drugs for migraine prophylaxis. Cochrane Database Syst Rev. 2004;(3):CD003226.

13. Welch KM, D'Andrea G, Tepley N, Barkley G, Ramadan NM. The concept of migraine as a state of central neuronal 


\section{JMSCR VoI||08||Issue ||07||Page 357-366||July}

hyper excitability. Neurol Clin. 1990;8(4):817-28.

14. Shank RP, Gardocki JF, Streeter AJ, Maryanoff BE. An overview of the preclinical aspects of topiramate: pharmacology, pharmacokinetics, and mechanism of action. Epilepsia. 2000;41 (Suppl 1):S3-S9.

15. Schurks M, Diener HC, Goadsby P. Update on the prophylaxis of migraine. Curr Treat Options Neurol. 2008;10 (1):20-9.

16. Guo Y, Han X, Yu T, Yao G. Metaanalysis of efficacy of topiramate in migraine prophylaxis. Neural Regen Res. 2012; 7(23): 1806-11.

17. Kinze S, Clauss M, Reuter U, Wolf T, Dreier JP, Einhäupl KM et al. Valproic acid is effective in migraine prophylaxis at low serum levels: a prospective open-label study. Headache. 2001;41(8):774-8.

18. Bartolini M, Silvestrini M, Taffi R, Lanciotti C, Luconi R, Capecci M, Provinciali. L Efficacy of Topiramate and Valproate in Chronic Migraine. Clinical Neuropharmacology.2005; 28(6):277-9.

19. Bavrasad R, Nejad S, Yarahmadi A, Sajedi S, Rahim F. Assessment of the Middle Dose of Topiramate in Comparison with Sodium Valproate for Migraine Prophylaxis: A Randomized-Double-Blind Study. International Journal of Pharmacology.2010; 6(5): 670-675. 